\title{
Circuit
}

Musiques contemporaines

\section{Culture numérique et électroacoustique}

\section{Alain Thibault}

Volume 13, numéro 1, 2002

L'électroacoustique : à la croisée des chemins?

URI : https://id.erudit.org/iderudit/902265ar

DOI : https://doi.org/10.7202/902265ar

Aller au sommaire du numéro

Éditeur(s)

Les Presses de l'Université de Montréal

ISSN

1183-1693 (imprimé)

1488-9692 (numérique)

Découvrir la revue

Citer cet article

Thibault, A. (2002). Culture numérique et électroacoustique. Circuit, 13(1),

51-56. https://doi.org/10.7202/902265ar

\section{Résumé de l'article}

Cet article questionne la place et les modes de représentation de la musique électroacoustique à l’intérieur de la culture numérique actuelle.
Ce document est protégé par la loi sur le droit d'auteur. L'utilisation des services d'Érudit (y compris la reproduction) est assujettie à sa politique d'utilisation que vous pouvez consulter en ligne.

https://apropos.erudit.org/fr/usagers/politique-dutilisation/ 


\title{
Culture numérique et électroacoustique
}

\author{
Alain Thibault
}

\begin{abstract}
L'art tendrait en somme aujourd'hui à occuper la place vide du théologiquepolitique et du rapport qu'il impliquait. [... A À partir du moment où elle [l'œuvre] n'est plus spectacle, mais événement, c'est-à-dire à partir du moment où elle n'est plus représentation de quelque chose mais participation à quelque chose, l'espace de l'œuvre se transforme radicalement en devenant lui-même l'ouverture à cette horizontalité de cette participation sur le fond sans fond du sens inappropriable ou, dit autrement, de la finitude.
\end{abstract}

Michel Gaillot'

II pouvait sembler radical en 1967 de s'asseoir dans une salle pour écouter des haut-parleurs alimentés par des bandes magnétiques diffusées en temps réel ${ }^{2}$. Issue du détournement des technologies du son, la musique électroacoustique apparaissait à l'époque comme LE mode d'expression musicale révolutionnaire. Cependant, les difficultés d'apprentissage de sa technique, plutôt artisanale à l'époque, l'inexistence d'un langage de composition proche de l'écriture instrumentale et le manque de stimuli visuels de son mode de représentation l'ont reléguée à un sous-genre de la musique contemporaine ${ }^{3}$. Par ailleurs, l'électroacoustique a toujours entretenu un rapport ambigu à la musique, plusieurs de ses praticiens et théoriciens ayant affirmé à maintes reprises que ce n'était justement pas de la musique mais un art différent, possédant un langage spécifique. Relation ambiguë également avec les nouvelles technologies, auxquelles on a recours pour leurs possibilités de création tout en conservant une forme de présentation issue des paramètres et valeurs du concert instrumental, lui-même façonné par les diverses figurations politiques et religieuses. Le concert avait probablement un sens en tant que situation de transition qui introduisait un nouveau genre musical auprès d'un auditoire possédant des habitudes d'écoute solidement ancrées. Ce mode de présentation, hérité de la période classique, semble de plus en plus inapproprié au contexte actuel d'accélération des changements technologiques que nous vivons, à la fois en musique électronique et plus largement dans tous les domaines. Cette évolution oblige à un ajustement constant de notre relation avec le temps et modifie nos capacités d'attention.
1. GAlLLOT, Michel, (1999), La techno. Un laboratoire artistique et politique du présent, Paris, Éditions Dis Voir, p. 59.

2. On peut situer à la fin des années 1960 le moment, en Europe, où les concerts de musique contemporaine instrumentale et électroacoustique suscitaient un engovement certain pour cette remise en question des langages musicaux et des modes de diffusion. Au Québec, cet élan des musiques contemporaines eut lieu quelques années plus tard, à la fin des années 1970. Cet intérêt a obligé alors les organismes gouvernementaux en charge de la culture à créer de nouveaux programmes et les institutions musicales à s'adapter à cette nouvelle situation en créant de nouveaux cours, en construisant des studios et en embauchant de nouveaux professeurs qui pouvaient enseigner ces nouvelles disciplines. II s'ensuivit une expansion du domaine, une multiplication des événements et concerts et du nombre de créateurs et d'interprètes. $L^{\prime} \propto$ institutionnalisation", au fil des ans, de la musique contemporaine a eu cependant comme effet négatif de retarder les remises en question.

3. L'électroacoustique n'a jamais réussi à rassembler autant de personnes que la musique instrumentale contemporaine. De ce fait, la majorité des subventions gouvernementales allouées à la diffusion de la nouvelle musique allait et va toujours aux ensembles instrumentaux. 
On dit souvent que les personnes qui grandissent dans un contexte de zappage télévisuel, de jeux vidéo et d'Internet ont une capacité d'attention plus restreinte que celle des générations précédentes. Pour les nouvelles générations, ce phénomène va sans doute encore s'accentuer puisque ses causes, loin de disparaître, vont en s'intensifiant. C'est l'état du monde maintenant et nous avons à vivre avec lui, tel qu'il est. Mais il importe également d'être conscient du côté positif que peuvent avoir ces changements individuels et ces nouveaux comportements. Ceux qui sont fortement exposés à cette culture semblent développer une nouvelle façon d'interagir avec leur environnement, beaucoup plus proche du multiprocessing ou du processing parallèle. Leurs processus de réception est sûrement moins linéaire que celui des générations précédentes. Ils sont davantage attirés par les expériences multisensorielles, interactives ou immersives que par les situations de type concerts, où ils ont le sentiment d'être gardés physiquement captifs. Pour eux, un événement modélisé de façon plus conviviale, qui permet le mouvement et un aller-retour de la concentration, est beaucoup plus naturel. On nous a appris à rejeter ce type d'événement plus proche de la fête que de la culture sérieuse, mais on oublie que c'était de cette façon qu'étaient présentées les pièces de théâtre à l'époque de Shakespeare ${ }^{4}$.

Le concert est une forme de représentation très particulière, intimement liée à la montée de la bourgeoisie qui a nourri le mythe d'une grande culture, dissociée des masses, de la culture populaire. S'est développée alors, de manière "emphatique", la notion d'objet d'art, d'œuvre vouée à la pérennité, création d'un héros individualiste, auteur, génie. Plus récemment, dans des domaines comme les arts visuels et les communications, a surgi une nouvelle pensée qui a mené à une réévaluation radicale de ces idées, reconnues comme étant surtout typiques des périodes classique et romantique. Ce qui a conduit à repenser les modes de diffusion en fonction des nouveaux schèmes de création en émergence ${ }^{5}$, qui impliquent la participation active de l'auditoire, par opposition à la forme de concert traditionnelle où le public est appelé à la contemplation passive des produits de la grande culture. Ce besoin de renouvellement de la forme et du cadre de la communication artistique est lié entre autres à deux facteurs, ou phénomènes, très forts : l'émergence de la culture techno, elle-même centrée sur la musique électronique et une utilisation spécifique de sa lutherie, qui a pour corollaire le développement d'un certain sens de la communauté ${ }^{6}$ et, au cœur même de cette évolution, la démocratisation, entendue comme accessibilité et convivialité, des outils numériques hardware et software. Les individus avec ou sans études musicales pouvaient donc désormais produire de la musique électronique sans avoir à passer à travers les structures et les filets des institutions, sans parler de leurs influences "esthétiques", afin d'avoir accès à un équipement jadis extrêmement dispendieux.

Au cours des années 1990, une nouvelle culture musicale underground a émergé, favorisée par la diminution des coûts de production des disques compacts. Ce phénomène a permis la prolifération de petites étiquettes spécialisées surtout en musique électronique de type minimaliste. De là sont nés des événements ou des festivals axés sur les arts électroniques ${ }^{7}$ présentant, dans leur volet musique, les
4. On songe également à une scène du film Amadeus de Milos Forman, soit la première de l'opéra la flute enchantée de Mozart.

5. Se référer ici au texte de Roland Barthes, "La mort de l'auteur".

6. Se référer à l'éditorial de Chantal Pontbriand, «Électrosons_Electrosounds», dans le numéro 107 de la revue d'arts visuels contemporains Parachute. D'ailleurs, il est intéressant de noter que dans ce numéro consacré au son électronique, on ne fait à peu près pas référence aux acteurs montréalais de la scène électroacoustique.

7. Entre autres, Ars Electronica à Linz en Autriche, Sonar à Barcelone, Transmediale à Berlin, V2 à Rotterdam, Avanto à Helsinki, Media Lounge et Mutek à Montréal, ISEA - International Symposium for Electronic Arts, Exit à Créteil. 
artistes attachés à ce courant minimaliste électronique. On remarque que la majorité de ceux-ci ont une formation en arts visuels et qu'ils n'ont pas eu à subir le poids culturel des institutions musicales lorsqu'ils en viennent à la musique électronique. Is ont une approche beaucoup plus conceptuelle et quelquefois politique, une façon d'aborder ce médium qui s'appuie rarement sur les notions musicales traditionnelles de complexité et de qualitée ${ }^{8}$. En d'autres mots, le succès de leur travail sonore ne repose pas uniquement dans le produit ou la pièce, mais dans sa relation avec la technologie numérique, sa conceptualisation et son lien avec une communauté. Tout cela est, dans un sens important, contextuel, une notion essentiellement postmoderne avec laquelle les électroacousticiens sont généralement peu familiers, contrairement aux artistes visuels qui, depuis environ 25 ans, ont développé des pratiques contextuelles comme l'installation.

Les artistes sonores provenant des arts visuels ou possédant une formation non musicale considèrent leur pratique comme représentative de, et contribuant à la culture numérique dans son sens le plus large ${ }^{9}$. De son côté, la musique électroacoustique est restée une pratique "régionale ${ }^{10}$ ", et ses relations avec cette nouvelle culture électronique en émergence sont demeurées jusqu'ici assez limitées; elle est peu perméable aux questionnements que ce nouveau courant suscite. On note qu'en Europe, l'électroacoustique est absente des festivals d'art électronique. Les changements effectués entre 1997 et 1998 par les organisateurs du prix Ars Electronica dans la catégorie Computer Music (diversification de la composition du jury et son changement d'appellation par Digital Musics) afin de l'ouvrir à un plus grand nombre de genres et d'esthétiques musicales illustrent assez bien le malaise qui s'était installé devant l'approche unidirectionnelle du monde électroacoustique "l.

Présentement, dans les institutions d'enseignement musical, on trouve relativement peu d'aspirants en composition électroacoustique. Un public très restreint, le peu de possibilités de diffusion et le manque d'intérêt des médias sont certainement des facteurs déterminants de cette défection. Par contre en arts visuels, tout le monde veut faire de la musique électronique ou de l'art sonore minimaliste. Ces courants sont perçus comme la fine pointe de la culture contemporaine et exercent un attrait de plus en plus important auprès du jeune public. Ils tendent à fusionner une culture expérimentale, plus intellectuelle, avec une culture possédant un pouvoir d'attraction plus large.

L'électroacoustique est née du détournement et d'une utilisation spécifique des technologies de l'audio. Ces dernières sont en évolution contante, modifiant et façonnant nos vies. Si elle veut conserver un statut d'art non figé, la musique électroacoustique doit remettre en question ses modes de représentation et explorer des formes de diffusion plus radicales et plus stimulantes, en relation avec la culture numérique contemporaine. Des événements comme la série Elektra ${ }^{12}$, produite par I'ACREQ (Association pour la création et la recherche électroacoustiques du Québec ${ }^{13}$ ) et présentée à I'Usine C (Montréal) depuis 1999, illustrent bien l'évolution d'une société de concert qui s'est adaptée aux changements en cours en
8. Notons que ce sont des conservateurs et des publications en arts visuels qui ont reconnu en premier et fait connaître les compositeurs minimalistes américains tels que Steve Reich, Philip Glass, Terry Riley, Laurie Anderson, Alvin Lussier, La Monte Young, Robert Ashley, Pauline Oliveros Ivoir Parachute n 107, p. 114 à 1 17).

9. Ces artistes ont d'ailleurs introduit une nouvelle forme de représentation : le concert laptop. Le retour à l'avant-scène du performeur ou de l'exécutant en temps réel donne une sorte de repère humain ou visuel aux spectateurs, contrairement à l'acousmaticien qui met son œuvre en avant-plan. Cette référence à la culture populaire, cette réapparition de l'idée de rock ou pop star comme véhicule de transmission, peut effectivement être considérée comme un retour à des valeurs dépassées ou réactionnaires. D'ailleurs, on questionne de plus en plus ce type de représentation : en effet, dans une situation où le spectateur est de toute façon passif, quel est l'intérêt réel de se retrouver assis par terre ou debout dans un club à regarder «opérer » un artiste, généralement éclairé par l'écran de son ordinateur portable. À la suite de la troisième édition en 2002 du festival Mutek, au moins deux journalistes ont écrit qu'il serait bon, pour des questions de confort, de revenir à la formule de la salle avec sièges pour les présentations à caractère plus expérimental.

10. Le concept de concert de haut-parleurs, principale forme de diffusion de la musique électroacoustique au Québec, ne s'est pas répandu dans le monde occidental et est resté confiné surtout à des villes comme Montréal, Paris, Bourges, Marseille, Birmingham (RU). Cette pratique de l'électronica est restée très blanche et très mâle, tout comme, du reste, ses artistes et son public. 11. Naut Humon (Sound Traffic Control de San Franciscol fut l'instigateur de ce changement, les compositeurs de musique électroacoustique étant, jusqu'à cette période, majoritaires au sein du jury de la catégorie Computer Music.

12. mmw.elektrafestival.ca

13. L'ACREQ est une société de concerts créée en 1978, qui s'est donné comme principal mandat la diffusion de la musique électroacoustique, sous toutes ses formes. 
répondant et en tentant d'aller au-delà ${ }^{14}$ des attentes des créateurs et du public. Devant l'émergence de cette culture nouvelle, il était impératif de transformer et de faire évolver la structure de diffusion qui joue, depuis 1978, un rôle prépondérant dans le développement de la musique électroacoustique au Québec, et d'explorer d'autres genres connexes comme le multimédia, la vidéo, l'animation numérique, la robotique, la performance. L'événement Elektra est devenu après trois ans d'existence une manifestation importante en musique et en art électronique, dont la programmation se caractérise par un large registre esthétique, le plus représentatif possible des tendances de l'espace culturel technologique actuel.

L'électroacoustique doit se dissocier au plus vite des institutions musicales traditionnelles. À ses débuts, le monde de la musique contemporaine en fut à la fois le catalyseur et le moteur, mais il est devenu maintenant son plus grand frein et paralyse son évolution. De plus, les différences tant dans les processus de production que dans l'esthétique imposent une telle distanciation de la musique instrumentale. l'électroacoustique est à mon sens plus proche des arts médiatiques que de la musique. Ce médium n'échappe pas au crossover entre les disciplines, qui a donné naissance à des œuvres transdisciplinaires et multidisciplinaires intégrant musique, vidéo, animations numériques, robotiques, performance, etc. Cependant, la résistance et la peur des électroacousticiens à se confronter au monde des arts médiatiques sont probablement les principaux facteurs qui retardent un tel mouvement, qui serait plus que souhaitable. La pratique électroacoustique née des voies tracées par Schaeffer ou Stockhausen est un genre musical ayant atteint un certain degré de maturité et de classicisme ${ }^{15}$, bien établi dans ses formes de représentation. A partir de ce constat, il s'agirait tout simplement $d^{\prime}$ imaginer une nouvelle appellation, plus englobante, exprimant mieux les nouvelles réalités et rendant pleinement compte du principe d'évolution perpétuelle lié aux développements technologiques.

Un immense remerciement à lynn Hughes pour les conseils et les commentaires prodigués lors de l'écriture de cet article.
14. En novembre 1994, I'ACREQ avait présenté, en collaboration avec Soft Image, une performance de l'Australien STELARC, en ouverture d'un des raves de la série Neksus qui avait lieu dans un bâtiment désaffecté situé dans le Vieux-Port de Montréal où la compagnie de multimédia Discreet s'est ensuite établie.
15. Un peu comme le jazz.

\section{Bibliographie}

GAlLOT, M. (1999), La techno. Un laboratoire artistique et politique du présent, Paris, Éditions Dis Voir. 

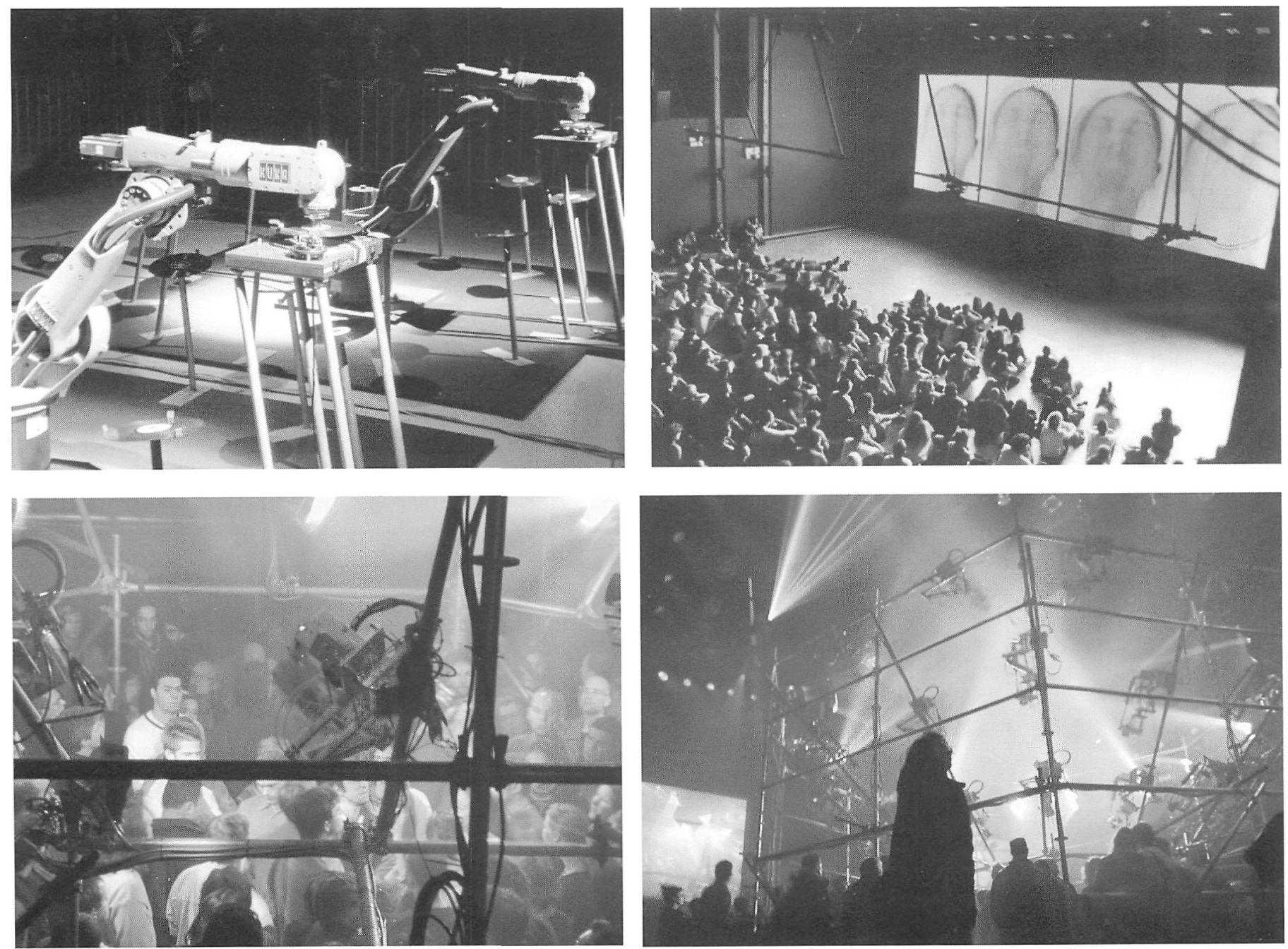

\section{Photos}

RobotLab (Mathias Gommel, Martina Haitz, Jan Zappe) - Juke Bots (Allemagne 2001 )

- Performance pour deux robots industriels et tourne-disque - ELEKTRA 2002.

Photo: RobotLab. wuw.robotlab.de/index_frz.htm

Granular Synthesis - Motion Control Modell5 (Ulf Langheinrich et Kurt Heinschläger Autriche version 1997) - Performance multimédia pour 4 écrans vidéo et musique électronique - ELEKTRA 2000. Photo: Brigitte Henry. wnw.thing.at/granular-synthesis/

Louis-Philippe Demers - L'assemblée (Canada/Allemagne 2001) - Performance robotique pour 48 membres robotiques, 48 haut-parleurs - ELEKTRA 2001. Photos : Peter

Dimakos. www. processing-plant.com 


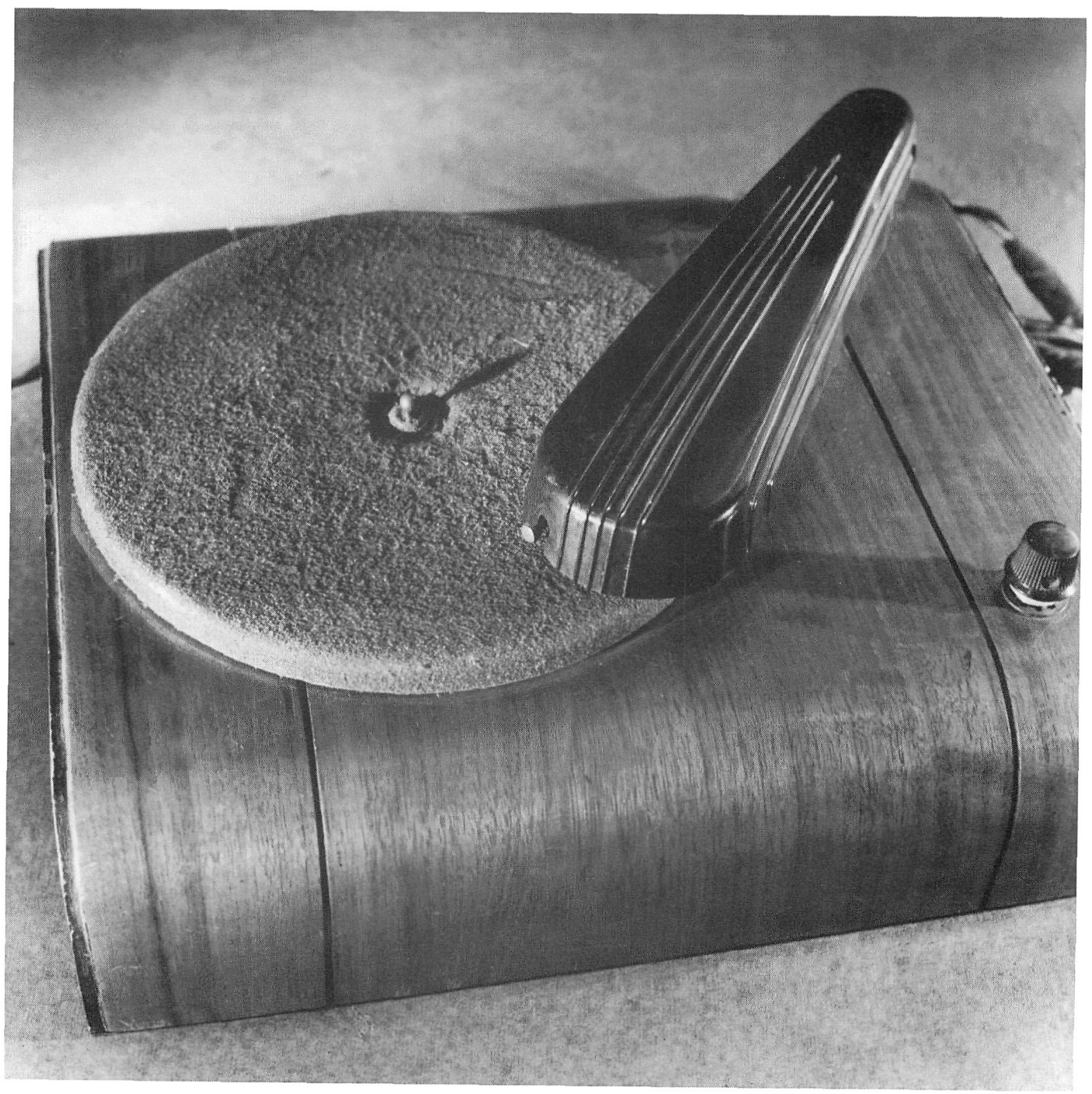

